\title{
Theta-modulated gamma-band synchronization among activated regions during a verb generation task
}

\author{
Sam M. Doesburg ${ }^{1,2,3}$, Sarah A. Vinette ${ }^{4}$, Michael J. Cheung ${ }^{1,2}$ and Elizabeth W. Pang ${ }^{2,5}$ \\ ' Department of Diagnostic Imaging, Hospital for Sick Children, Toronto, ON, Canada \\ ${ }^{2}$ Neurosciences and Mental Health Program, Hospital for Sick Children Research Institute, Toronto, ON, Canada \\ ${ }^{3}$ Department of Medical Imaging, University of Toronto, Toronto, ON, Canada \\ ${ }^{4}$ Department of Neuroscience, University of Calgary, Calgary, AB, Canada \\ ${ }^{5}$ Department of Neurology, Hospital for Sick Children, Toronto, ON, Canada
}

\section{Edited by:}

Lucia Melloni, Max Planck Institute for Brain Research, Germany

\section{Reviewed by:}

Stefano F. Cappa, Vita-Salute San

Raffaele University, Italy

Dirk Koester, Bielefeld University, Germany

Juan R. Vidal, Institut National de la Santé et de la Recherche Médicale, France

\section{*Correspondence:}

Sam M. Doesburg, Department of Diagnostic Imaging, The Hospital for Sick Children, 555 University Avenue, Toronto, ON, Canada M5G 1 X8 e-mail: sam.doesburg@sickkids.ca
Expressive language is complex and involves processing within a distributed network of cortical regions. Functional MRI and magnetoencephalography (MEG) have identified brain areas critical for expressive language, but how these regions communicate across the network remains poorly understood. It is thought that synchronization of oscillations between neural populations, particularly at a gamma rate $(>30 \mathrm{~Hz})$, underlies functional integration within cortical networks. Modulation of gamma rhythms by theta-band oscillations $(4-8 \mathrm{~Hz})$ has been proposed as a mechanism for the integration of local cell coalitions into large-scale networks underlying cognition and perception. The present study tested the hypothesis that these oscillatory mechanisms of functional integration were present within the expressive language network. We recorded MEG while subjects performed a covert verb generation task. We localized activated cortical regions using beamformer analysis, calculated interregional phase locking between activated areas, and measured modulation of inter-regional gamma synchronization by theta phase. The results show task-dependent gamma-band synchronization among regions activated during the performance of the verb generation task, and we provide evidence that these transient and periodic instances of high-frequency connectivity were modulated by the phase of cortical theta oscillations. These findings suggest that oscillatory synchronization and cross-frequency interactions are mechanisms for functional integration among distributed brain areas supporting expressive language processing.

Keywords: expressive language, neural oscillation, neural synchrony, cross-frequency, magnetoencephalography, beamformer, functional connectivity, neural network, theta-band, gamma-band

\section{INTRODUCTION}

Expressive language refers to the cognitive processes involved in language production and transmission. While it is usually oral, it can take written and gestural forms as well. Experimental manipulations of expressive language usually require subjects to name an object, generate a verb, or read a word. Although these are simple tasks, they still require the coordination of a number of cognitive processes such as word selection, word retrieval from memory, syntactic and phonological encoding, articulation, and auditory feedback (Levelt et al., 1999). This seemingly simple phenomenon is in reality very complex and involves multiple specialized cortical regions. A recent review (Price, 2010) of hemodynamic studies of both receptive and expressive language reported superior, middle, and inferior temporal cortices, left angular gyrus and left pars orbitalis, for both types of language, with additional involvement of left middle frontal cortex, left anterior insula, anterior cingulate, and subcortical areas for speech production. While classical models of language (e.g., Geschwind, 1970) have linked distinct brain areas with specific functions, current research models (e.g., Hickok and Poeppel, 2004; Indefrey and Levelt, 2004; Golfinopoulos et al., 2010) have shifted focus away from ones that segregate language function to ones that explain how disparate neural areas are integrated to produce function.

Studies of structural connectivity, primarily conducted using diffusion tensor imaging (DTI), have explored network connections among known language areas (Catani et al., 2005; Frey et al., 2008; Saur et al., 2008; Ford et al., 2010; Turken and Dronkers, 2011). These DTI studies, along with other structural connectivity studies (e.g., Kelly et al., 2010), have provided a strong understanding of the neuroanatomy of language networks. However, structural connectivity studies alone cannot account for functional interactions among brain areas implicated in language. Studies of resting-state functional connectivity in language show that strong regional coupling is related to reading ability (Koyama et al., 2011) and that short-range connectivity dominates within the language network, consistent with a reliance on local processing, with some left-lateralized long-range connectivity. Connections between language and sensory areas, however, have been shown to be anti-correlated suggesting that they operate sequentially (Tomasi and Volkow, 2012). Although fMRI has been used to examine the functional connectivity of receptive language areas (Friederici et al., 2011), expressive language has not yet been 
studied. To date, the mechanisms underlying task-dependent functional connectivity within the expressive language network remain poorly understood.

Neural synchronization has been proposed as a mechanism underlying the formation of task-specific functional networks (Varela et al., 2001), as synchronously oscillating neurons exert greater influence on one another's activity (Fries, 2005). The veracity of this insight has been borne out by studies comparing inter-regional synchronization with causal interactions and mutual information (Supp et al., 2007; Womelsdorf et al., 2007), and evidence linking phase relations among oscillations in widely distributed cortical regions to the behavior of single neurons and the formation of Hebbian constellations of co-active neurons (Canolty et al., 2010). Early studies into the role of neural synchrony in the formation of functional cell assemblies supporting perceptual integration identified gamma-band oscillations centered around $40 \mathrm{~Hz}$ as critical (Gray and Singer, 1989; Gray et al., 1989; Engel et al., 1991). Subsequent invasive and noninvasive studies using humans have confirmed the relevance of inter-regional gamma-band synchronization in cognition and perception (Sarnthein et al., 1998; Rodriguez et al., 1999; Doesburg et al., 2005, 2008a, 2009, 2012; Rose and Büchel, 2005; Rose et al., 2006; Buschman and Miller, 2007; Siegel et al., 2008), including receptive language (Weiss et al., 2005), language learning (de Diego-Balaguer et al., 2011), and audio-visual speech segregation (Doesburg et al., 2008b).

Gamma activity within the expressive language network has been examined using electrocorticography (ECoG). The high temporal and spatial resolution of ECoG allows tracking of gamma activity to map the spatiotemporal dynamics of cortical networks supporting the performance of expressive language tasks with varying levels of linguistic complexity; for example, phoneme repetition (Fukuda et al., 2010), word repetition (Pei et al., 2011), verb generation and picture naming (Edwards et al., 2010), and word production (Crone et al., 2001). To understand the role of gamma activity in functional network connectivity, one study applied event-related causality analyses to ECoG recordings and found prominent causal interactions between fronto-temporal language areas and motor control areas which correlated with task parameters (Korzeniewska et al., 2011).

Low-frequency oscillations have been purported to integrate gamma-oscillatory neuronal coalitions into large-scale network dynamics encompassing activity across disparate brain regions (von Stein and Sarnthein, 2000). Interplay among gamma and theta oscillations may thus play a pivotal role in the representation and transfer of information within the cortex (see Ward, 2003; Canolty and Knight, 2010), and mediate the selective functional integration and segregation of neuronal assemblies (Llinás et al., 2005). Gamma-band activity has been shown to be modulated by theta rhythms during cognitive and perceptual processing (Schack et al., 2002; Canolty et al., 2006; Demiralp et al., 2007; Sauseng et al., 2008) and modulation of inter-regional gamma-band connectivity by cortical theta oscillations has also been demonstrated during perception (Doesburg et al., 2009) and cognition (Burgess and Ali, 2002; Doesburg et al., 2012). Such observations lead to the hypothesis that expressive language processing entails theta-modulated gamma-band synchronization among activated task-relevant cortical regions.

To test the hypotheses that inter-regional gamma synchrony, reflecting functional integration, is increased among task-relevant brain regions during expressive language processing, and that these inter-regional interactions were mediated by cortical theta rhythms, we recorded magnetoencephalographic (MEG) activity from healthy controls during the performance of a covert verb generation task. To determine seed points for the synchronization analysis, beamformer analysis was used to localize areas expressing activation relative to a pre-stimulus baseline, phase synchronization between these nodes was calculated and modulation of inter-regional gamma-band synchronization by theta phase was assessed. Rather than exploring the neural basis of specific domains of language processing, such as semantics, phonology, and syntax, this approach investigates how multiple specialized brain regions coordinate oscillatory activity to support expressive language processing. MEG recordings are ideal for this purpose as they are a neurophysiological measure, obtained over the wholehead, and offer an excellent combination of high spatial and temporal resolution. This research builds upon previous investigations of the temporal dynamics of receptive language, and to some extent, expressive language, using MEG (see Salmelin, 2007, for a review), as well as a covert verb generation paradigm recently developed for localization of areas within the expressive language network (Kadis et al., 2011; Pang et al., 2011). This task was based on a classical fMRI paradigm used in clinical settings (Holland et al., 2001; Gaillard et al., 2003; Wood et al., 2004; Brown et al., 2005), and has been validated against the intracarotid sodium (IAP) test (Szaflarski et al., 2008) and direct cortical stimulation (Roux et al., 2003). The use of verbs and nouns recently have been demonstrated to activate similar frontal areas (left medial and inferior frontal; BA 9/10 and 45) and bilateral cingulate cortex with verbs further activating left middle temporal gyrus (Khader et al., 2010), although left inferior frontal cortex seems to be more robustly activated with verbs (Perani et al., 1999; Khader and Rösler, 2004; Shapiro et al., 2006). Both the noun and verb generation tasks share similar processes of word selection, retrieval, phonological encoding, and articulation (Edwards et al., 2010; Schuhmann et al., 2012). For these reasons, we chose to use the clinically validated verb generation task and asked adolescent participants to perform covert verb generation in the MEG.

\section{MATERIALS AND METHODS SUBJECTS}

Thirteen subjects (six females; mean age $=17.7 \pm 1.7$ years) participated in the study. All subjects were right handed, assessed using the Edinburgh Handedness Inventory (mean = 85.7 \pm 11.63 ; Oldfield, 1971), and gave written informed consent. This study was approved by the Hospital for Sick Children Research Ethics Board and conformed with the conventions set out in the Declaration of Helsinki. Previous research using the methods employed in the present investigation has indicated that task-dependent changes in local oscillatory activity are of considerably greater magnitude than task-dependent changes in inter-regional synchrony (Doesburg et al., 2008a,b, 2009), placing signal-to-noise demands on the analysis of inter-regional phase locking. Accordingly, noisy data 
from three subjects were excluded from the connectivity analyses. The resultant group consisted of five females and five males (mean age $=17.5 \pm 1.08)$.

\section{VERB GENERATION TASK}

Subjects performed a covert verb generation task, in English, within the MEG, in which color drawings of a set of common objects were presented on a screen (Figure 1). The full list of words presented is contained in Table 1. The list of objects was developed in consultation with standardized language batteries (e.g., Peabody Picture Vocabulary Test, Expressive Vocabulary Test, MacArthur Communicative Development Inventory) so that all objects were familiar to typically developing 5 year olds (which is a requirement for our clinical application of this paradigm), and included objects such as a ball, bicycle, apple, chair, broom, knife, net, hammer, etc. As these were all very familiar items, our adult subjects could quickly and easily generate single syllable verbs, e.g., throw, ride, eat, for the preceding examples. Subjects were instructed to silently and rapidly think of a single action word associated with that image (see Kadis et al., 2011; Pang et al., 2011). Images were presented for $500 \mathrm{~ms}$, followed by a baseline stimulus for $2000 \mathrm{~ms}$.

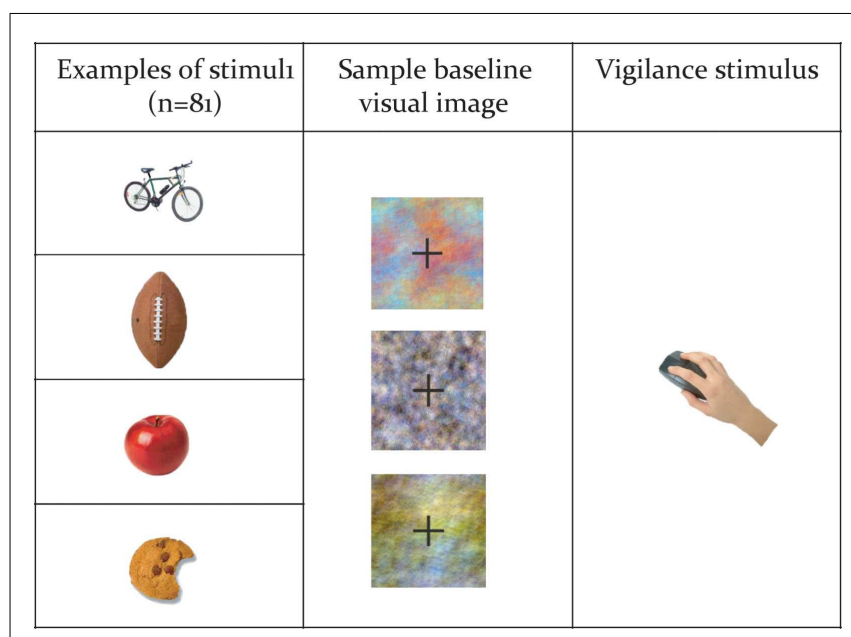

FIGURE 1 | Examples of stimuli from the covert verb generation task.
Baseline stimuli consisted of color-frequency scrambled images created from the pictures and matched for size, contrast, and luminance (see Figure 1). The experimental protocol comprised 92 trials. There were 81 stimulus trials. Vigilance trials, which consisted of a picture of hand pressing a button, were presented on $12 \%$ of trials to monitor subject attention. Subjects were instructed to press a button on a keypad on vigilance trials, and repeated this task outside the scanner to ensure compliance and performance. Vigilance trials were excluded from further analyses.

\section{DATA ACQUISITION}

MEG data were acquired using a 151 channel whole-head system (CTF Omega, Port Coquitlam, Canada) at $625 \mathrm{~Hz}$ and low-pass filtered at $200 \mathrm{~Hz}$ while subjects performed the covert verb generation task. Data were epoched from $-500 \mathrm{~ms}$ pre-stimulus to $1300 \mathrm{~ms}$ poststimulus on each experimental trial. Similar epoch lengths have previously been shown to be sufficient to capture network activation and inter-regional synchronization underlying cognition, perception, and language processing (Doesburg et al., 2008a,b, 2012). In order to co-register functional MEG data with neuroanatomy, structural (3D SPGR T1-weighted) MRI scans were collected from all subjects using an Excite HD 1.5 T MRI system (GE Medical Systems, Waukesha, WI).

\section{BEAMFORMER SOURCE LOCALIZATION}

To define seed points for the connectivity analysis based on changes in focal brain activity within the present dataset beamformer analysis was performed. Activity in multiple non-overlapping time windows relative to stimulus onset $(0-200,200-400,500-600$, $600-800 \mathrm{~ms})$ were contrasted with activity during a pre-stimulus baseline ( -250 to $-50 \mathrm{~ms}$ ). This differential beamformer analysis was carried out in multiple functionally relevant frequency ranges $(5-15,15-25,25-50,50-80 \mathrm{~Hz})$ using synthetic aperture magnetometry (SAM; Van Veen et al., 1997; Robinson and Vrba, 1999; Sekihara et al., 2001; Vrba and Robinson, 2001). This approach has been demonstrated to accurately represent the activity of cortical generators even in the presence of eye movements (Cheyne et al., 2006). The results of the beamformer analysis were coregistered to each subject's volumetric MR and normalized to a MNI template brain (Mazziotta et al., 1995, 2001) using AFNI (http://afni.nimh.nih.gov), and averaged across participants. The

Table 1 | List of stimuli (in alphabetical order) used for verb generation task.

\begin{tabular}{|c|c|c|c|c|c|c|}
\hline Anchor & Apple & Baby & Ball & Balloons & Baseball & Bat \\
\hline Bed & Bee & Bell & Bench & Bicycle & Bird & Boot \\
\hline Bottle & Bread & Broom & Carrot & Cat & Chair & Clown \\
\hline Comb & Crab & Cub & Dinosaur & Dog & Door & Ear \\
\hline Fan & Fish & Flashlight & Football & Frog & Glove & Hair \\
\hline Hammer & Hand & Hanger & Helicopter & Horse & Kangaroo & Key \\
\hline Knife & Light & Lion & Motorcycle & Mouse & Net & Paintbrush \\
\hline Palette & Pear & Pen & Pencil & Piano & Pig & Pipe \\
\hline Plant & Rabbit & Rake & Rope & Scissors & Shark & Ship \\
\hline Shirt & Shoe & Snake & Sock & Spoon & Suitcase & Cell phone \\
\hline Television & Tiger & Tire & Toaster & Tomato & Train & Truck \\
\hline Turtle & Wheel & Whistle & Windmill & & & \\
\hline
\end{tabular}

High quality color drawings were obtained for each word. 
results of the SAM beamformer analysis were screened for activation within the cerebral cortex. Beamformer analysis of highfrequency activity $(25-50$ and $50-80 \mathrm{~Hz}$ ) showed only cerebellar and brainstem sources for most time windows. In the lower frequency windows (5-15 and $15-25 \mathrm{~Hz}$ ), all activations were in the cerebral cortex. Accordingly, for each of these frequency bands, at each time window, the location with the highest pseudo-z score was selected as showing task-dependent activation.

\section{INTER-REGIONAL PHASE SYNCHRONIZATION}

Broadband time series representing activity within each activated region were extracted using event-related beamforming (Cheyne et al., 2006). This technique implements a spatial filter that estimates the activity at a desired location in source space through a weighted sum of sensor recordings in which the activity at all other locations is maximally attenuated without any change in power at the reconstructed source location (Sekihara et al., 2001). Reconstructed broadband time series representing each activated source were then filtered from $10-60 \mathrm{~Hz}$ at $1 \mathrm{~Hz}$ intervals (passband $=f \pm 0.05 f$, where $f$ represents the filter frequency). We then calculated the analytic signal

$\varsigma(t)=f(t)+i \tilde{f}(t)=A(t) e^{i \phi(t)}$

of the filtered waveform for each epoch $f(t)$, where $\tilde{f}(t)$ is the Hilbert transform of $f(t)$ and $i=\sqrt{-1}$, to obtain the instantaneous phase, $\varphi(t)$, and amplitude, $A(t)$, at each time point. Synchronization among activated brain regions was measured using phase locking values (PLVs). PLVs were calculated from instantaneous phase differences between reconstructed time series from pairs of sources, for example, sources $j$ and $k$, at each time point, $t$, across $N$ epochs (Lachaux et al., 1999):

$P L V_{j, k, t}=N^{-1}\left|\sum_{N} e^{i\left[\phi_{j}(t)-\phi_{k}(t)\right]}\right|$

To indentify changes in inter-regional synchrony associated with expressive language processing, we standardized PLVs relative to a $200 \mathrm{~ms}$ pre-stimulus baseline period by subtracting the mean baseline PLV, at that frequency, from each time-frequency point, and dividing this result by the standard deviation in PLVs during the baseline period.

Surrogate statistical methods were employed to assess the reliability of changes in inter-regional phase synchronization (see Lachaux et al., 1999). This was accomplished by scrambling the epochs and computing PLVs for the scrambled data for each timefrequency point, for each pair of sources. The resultant surrogate PLV time series was then standardized relative to the scrambled $200 \mathrm{~ms}$ baseline period. This was repeated 200 times to create a surrogate distribution for the relevant standardized PLV. This statistical analysis was limited to 200 surrogates as creation of a surrogate distribution was required for each time-frequency point, which imposed computational limitations. Percentile rankings of real PLV data within the surrogate distribution were used to assess statistical significance ( $p<0.05$; two tailed), wherein real PLVs above the 97.5th percentile were considered significant synchronizations and real PLVs below the 2.5 th percentile were considered significant desynchronizations. In addition, to control for false positives due to multiple comparisons, we also required that in order for a given instance of synchronization/desynchronization to be considered significant it would have to meet the surrogate statistical threshold for at least four consecutive time points and four consecutive frequency points.

CROSS-FREOUENCY MODULATION OF INTER-REGIONAL CONNECTIVITY To investigate the hypothesis that inter-regional gamma-band synchrony is modulated by the phase of cortical theta oscillations, we obtained the epoched time series of instantaneous phases of 40 and $6 \mathrm{~Hz}$ rhythms for each activated region using the Hilbert transform, as described above. These frequencies were selected as they are centered within theta and gamma-ranges reliably implicated in cognitive processing, to maintain consistency with signal processing methods employed for the analysis of inter-regional phase locking, and in order to employ established methods for characterization of cross-frequency modulation of inter-regional synchronization (see Doesburg et al., 2009, 2012). Gamma-band phases from a given pair of sources were then sorted according to theta phase into 60 bins of 0.105 radians width, and mean PLV was calculated within each bin, producing an index of the magnitude of gamma-band phase locking between a pair of activated regions during a particular phase of theta oscillations in one of the sources comprising that pair. Surrogate statistics were employed to assess the reliability of cross-frequency modulation of interregional phase locking (Doesburg et al., 2009, 2012), which are analogous to the statistical methods used for assessment of PLV changes, except that phases are shuffled with respect to theta phase rather than across trials. To this end, we shuffled the data and calculated gamma-band PLV within each bin. This process was repeated 1000 times, producing a surrogate distribution for each bin. We considered modulation of inter-regional synchronization to be statistically significant ( $p<0.05$; two tailed) if the measured PLVs were above the 97.5 th percentile, or below the 2.5 th percentile, of the surrogate distribution in at least four consecutive bins.

\section{RESULTS}

\section{LOCALIZATION OF THE EXPRESSIVE LANGUAGE NETWORK}

Beamformer source localization revealed task-dependent increases in low-frequency power $(5-15 \mathrm{~Hz} ; 15-25 \mathrm{~Hz})$ in distributed cortical areas: left inferior and medial frontal gyri, left orbital gyrus, left lingual gyrus, right insula, and right inferior and medial frontal gyri. All activated regions, associated Brodmann areas and Talairach coordinates are presented in Table 2 . These brain regions are consistent with previous neuroimaging literature on expressive language (Salmelin, 2007; Price, 2010), and provided the seed points for the synchronization analysis based on regional power changes within the present data.

\section{GAMMA-BAND SYNCHRONIZATION AMONG ACTIVATED REGIONS}

Task-dependent increases in gamma-band synchronization were observed between multiple source pairs within the activated expressive language network. Observed instances of inter-regional synchronization were transient and periodic, recurring at a rate consistent with a theta oscillation (Figures 2A,B). Inter-regional gamma synchronization was most abundant within the left hemisphere, particularly between left inferior frontal gyrus and other 
Table 2 | Each region exhibiting task-dependent activation and its associated Brodmann area and Talairach coordinates (IFG, inferior frontal gyrus; MFG, medial frontal gyrus).

\begin{tabular}{llccc}
\hline Region & Brodmann area & X & Y & Z \\
\hline Left IFG & 46 & -35 & 39 & 7 \\
Left IFG & 10 & -45 & 44 & -2 \\
Left MFG & 10 & -20 & 49 & 7 \\
Left orbital gyrus & 11 & -5 & 47 & -23 \\
Left lingual gyrus & 18 & 0 & -83 & -13 \\
Right insula & 13 & 40 & 19 & -1 \\
Right MFG & 47 & 45 & 34 & -6 \\
Right IFG & 47 & 45 & 24 & -14 \\
\hline
\end{tabular}

regions in left frontal cortex (Figure 2C). Little task-dependent gamma synchronization was observed within activated regions of right frontal cortex, or between left and right frontal lobes. Gamma-band synchronization between visual cortical areas and frontal regions in both left and right cerebral hemispheres was observed, and was particularly pronounced between left lingual gyrus and left inferior frontal gyrus. A schematic representation of network synchronization during expressive language processing is depicted in Figure 3. No systematic network synchronization effects were observed outside the gamma frequency range.

To investigate the spatial specificity of inter-regional gammaband synchronization we constructed a surrogate network of cortical seed points. Since left IFG (BA 10) emerged as a strongly connected hub in the expressive language network (see Figure 2C) we maintained this source at its "real" location and investigated phase locking between this source and other surrogate locations. Surrogate cortical locations were generated by shifting the $x, y$, and $z$ Talairach coordinates an equal distance from left IFG as the real source locations. In the case of midline and right hemispheric sources the $x$ coordinate was not shifted. In cases where this manipulation would cause the surrogate seed point to deviate from the gray matter the $x$ coordinate was adjusted so that the surrogate seed point was within the nearest gray matter. Gammaband $(40 \mathrm{~Hz})$ phase locking between left IFG (BA 10) and all other sources in the network was then compared between the real source montage and the surrogate sources. As previous studies have reported periodic and transient instances of gamma-band synchronization, interposed by periods of quiescence, or taskdependent desynchronization (i.e., Rodriguez et al., 1999; Doesburg et al., 2005, 2008b; Canolty and Knight, 2010), consistent with the results of the present study (see Figures 2A,B), we calculated the absolute value of the normalized PLV at each time point and averaged across all values within the analyzed epoch $(0-1000 \mathrm{~ms})$. This analysis revealed that task-dependent changes in gamma synchrony trended toward being greater in the real network than the surrogate network, although this effect did not reach statistical significance $(p=0.32)$. Comparison of real and surrogate gamma synchrony between left IFG (BA 10) and left MFG, where taskdependent changes were most robust (see Figure 2C), indicated that gamma synchrony among the real sources was higher than their surrogate counterparts $(p=0.017)$.

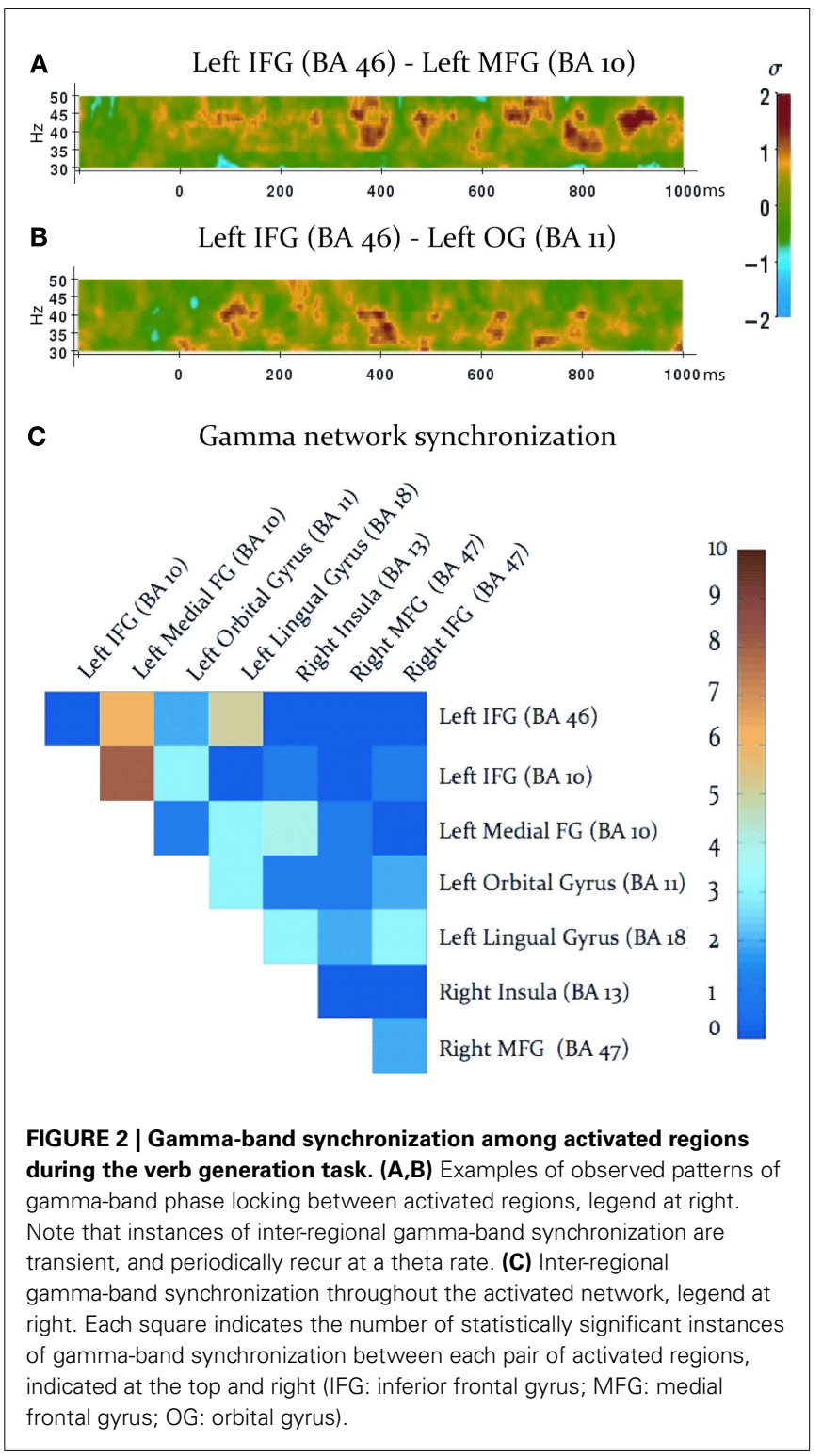

To determine if observed synchronization effects could be attributed to volume conduction the distribution of instantaneous phase differences among analyzed sources pairs was examined. Since volume conducted signals would arrive instantaneously at both locations, removal of zero-phase-lag synchronization is considered to be an effective safeguard against spurious synchronization due to volume conduction (i.e., Stam et al., 2007; Calmels et al., 2012; Hillebrand et al., 2012). A drawback of this approach is that physiological zero-phase-lag coherence is known to exist in the brain and be relevant for neural function (see Uhlhaas et al., 2009 for review). To quantify the degree to which volume conduction may have influenced our results without removing the record of real neural interactions, we examined the proportion of gammaband phase difference among all analyzed source pairs which occurred with zero-phase-lag throughout the analyzed epoch (0$1000 \mathrm{~ms}$ ). This revealed that, on average, only $2.7 \%$ of phase differences were zero-phase-lag across all analyzed pairs. Among 


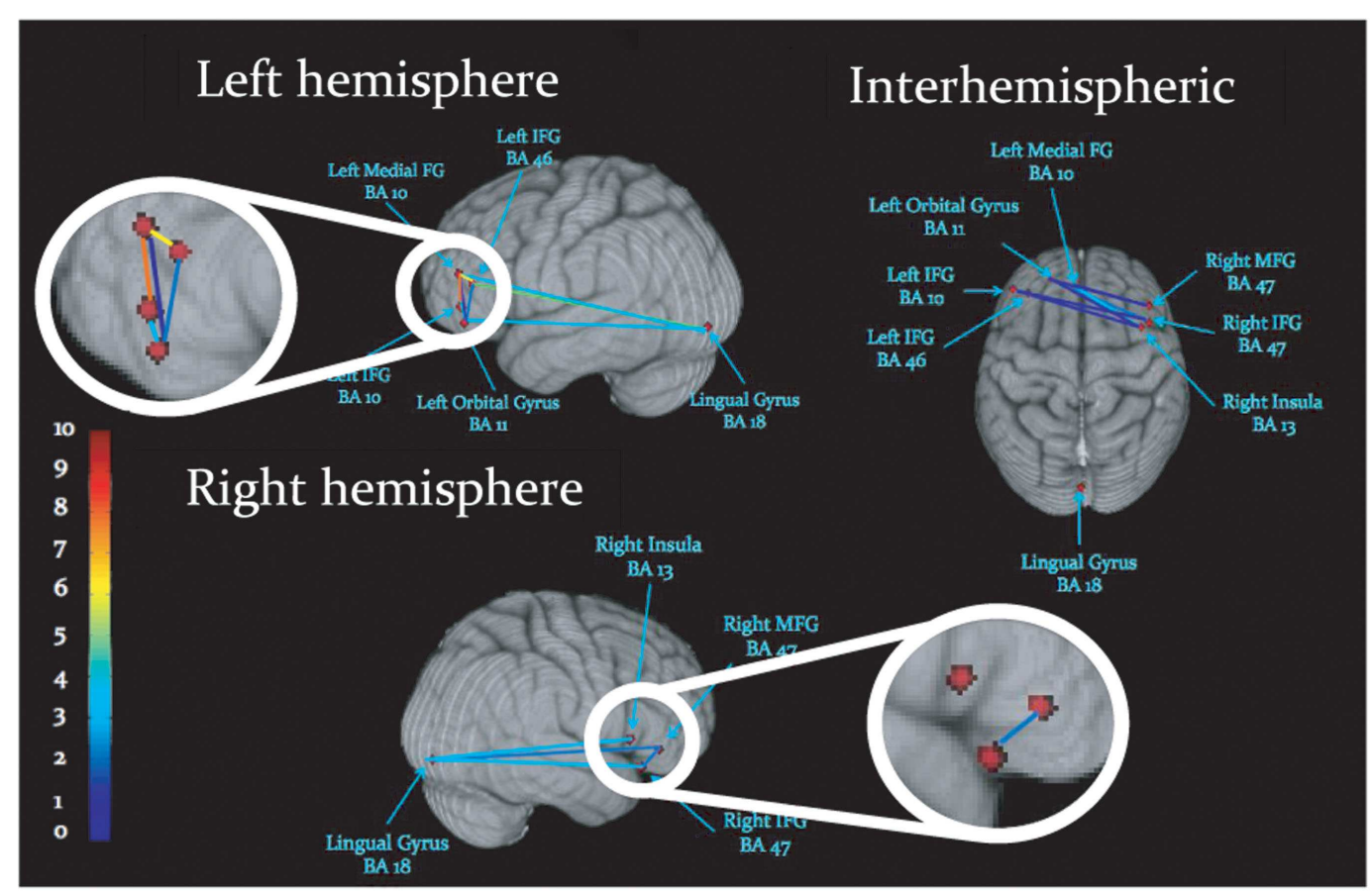

FIGURE 3 | Schematic display of inter-regional synchronization, where dots represent peaks of cortical activation, and lines reflect gamma-band synchronization among activated regions. Colors reflect the number of statistically significant synchronizations, legend at left.

pairs in the left frontal cortex, where abundant inter-regional activations were observed, instances of zero-phase-lag differences were slightly higher at $4.9 \%$, but still minimal. Importantly, zero-lag phase differences were lower between left IFG (BA 10) and left MFG, which showed the most pronounced gamma synchronization, than for left frontal cortex in general, indicating that the relationship between increased phase locking and zero-lag phase differences are not systematic. Furthermore, the time courses of inter-regional gamma-band synchronizations were not related to those of the source amplitudes, as would be expected in the case of synchronization arising from volume conduction. The abundant gamma-band synchronization between left MFG (BA 10) and left IFG (BA 10), for example, was not correlated with changes in gamma amplitude in either source $(r=0.07$ and $r=0.05$, respectively). Further pursuant to this point, no cortical activations were observed in the gamma-band during source localization (e.g., $25-50 \mathrm{~Hz}, 50-80 \mathrm{~Hz}$ bins), precluding a strong oscillating source which could impose volume conducted synchrony among brain regions. For these reasons, the synchronization results presented here are not attributable to volume conduction among nearby sources.

\section{MODULATION OF INTER-REGIONAL GAMMA SYNCHRONY BY THETA PHASE}

Gamma-band phase locking among activated brain regions was found to be modulated by the phase of cortical theta oscillations. The specific relationship between theta phase and inter-regional gamma phase locking differed among source pairs, in that the maxima and minima of gamma PLV could occur at different phases of the theta cycle (Figures 4A,B). Theta modulation of inter-regional gamma synchrony was observed between many pairs of activated sources (Figure 4C), but was most prevalent among sources in left frontal cortex. Some cross-frequency modulation of interhemispheric frontal synchronization was observed, and tended to be modulated by the theta phase in the left hemispheric source rather than the right. Cross-frequency modulation of gamma synchrony was found between visual cortex and frontal sources in both the left and right frontal lobes. Instances were found where gamma phase locking was modulated by the theta phase of both sources, although more commonly gamma synchrony was modulated by theta phase in only one of the source pairs.

\section{DISCUSSION}

Beamformer analysis of focal changes in power during covert expressive language processing localized a distributed network of cortical areas congruent with neural regions known to be involved in expressive language (Salmelin, 2007; Price, 2010), providing seed locations for the synchronization analysis based on regional activity changes within the present data. Cortical foci identified using beamformer analysis revealed task-dependent changes in low-frequency activity $(5-15 \mathrm{~Hz} ; 15-25 \mathrm{~Hz})$. Changes in cortical activity were likely observed within the lower frequency bands as changes in such activity typically encompasses a significantly more expansive area than coincident gamma activations (Jerbi et al., 2009). These results do not likely indicate a privileged relationship between local power changes in the beta-band and long-range gamma synchronization, but rather reflect the increased sensitivity of low-frequency power analysis to focal activations associated 


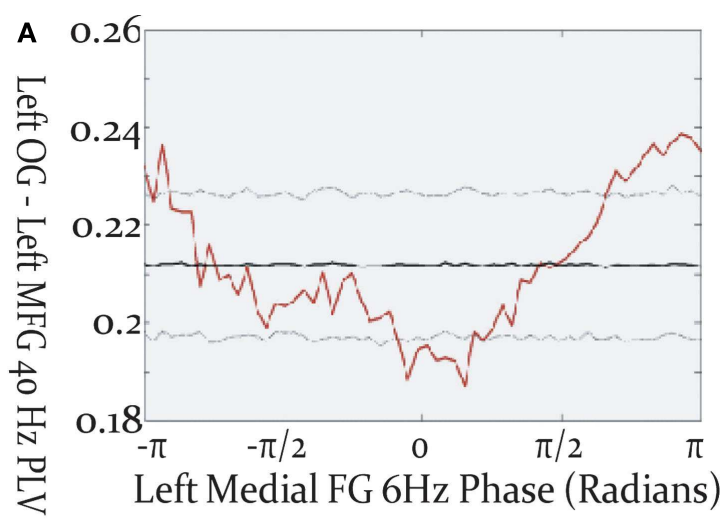

B
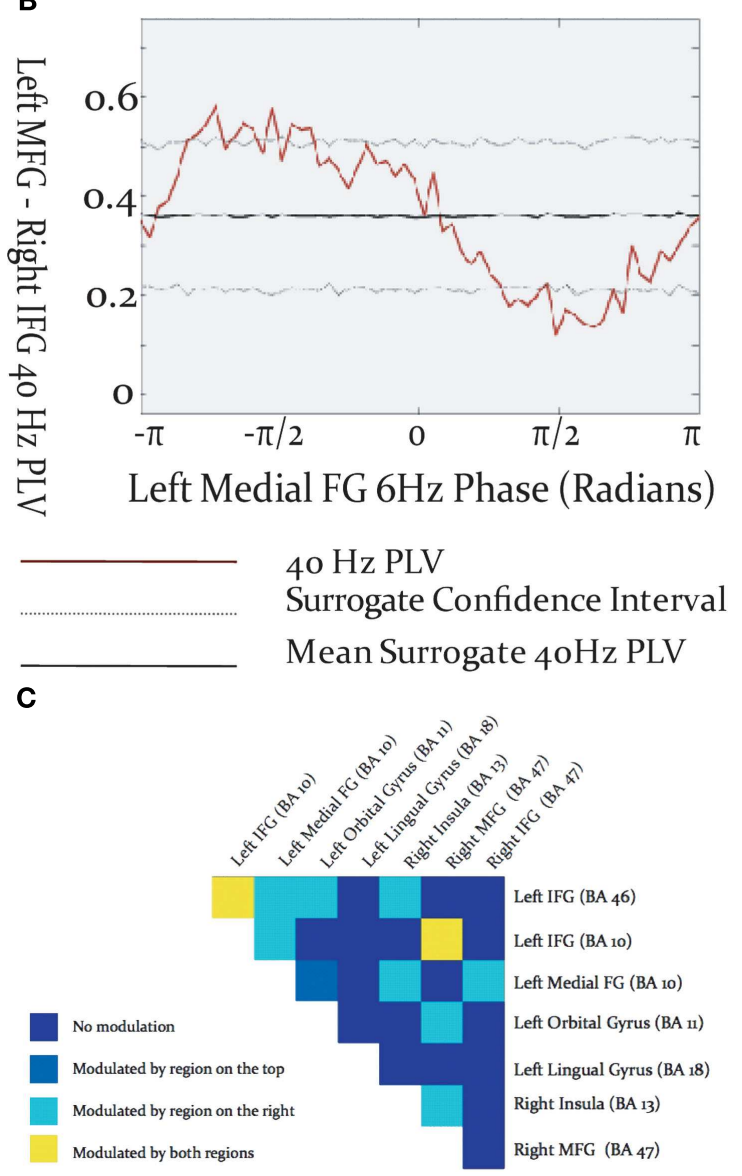

FIGURE 4 | Modulation of inter-regional gamma-band synchronization by theta phase within the activated network. $(A, B)$ Examples of cross-frequency modulation of inter-regional synchrony. (C) Theta modulation of inter-regional gamma synchronization throughout the activated network. Each box represents a region pair, listed at lop and right, and colors reflect whether gamma-band phase locking was modulated by theta phase in one, both, or neither of the regions.

with expressive language processing, as previous findings have indicated that local changes in MEG oscillatory activity during language processing are most pronounced in the beta frequency range (i.e., Hirata et al., 2010; Wang et al., 2011). The results presented here are congruent with the view that language processing likely involves activation in multiple brain regions which interact to produce language function (Hickok and Poeppel, 2004; Golfinopoulos et al., 2010; Friederici et al., 2011).

The present study provides the first evidence of task-dependent inter-regional phase synchronization within the expressive language network. Synchronization among activated regions in the gamma frequency range implicates integrative mechanisms similar to those reported in other domains such as working memory (Sarnthein et al., 1998), selective attention (Buschman and Miller, 2007; Doesburg et al., 2008a; Siegel et al., 2008; Gregoriou et al., 2009), and perception (Rodriguez et al., 1999; Doesburg et al., 2005, 2009; Rose and Büchel, 2005; Rose et al., 2006; Melloni et al., 2007). This consistent implication of gamma-band phase locking among cortical regions during task-related processing across cognitive contexts likely explains why robust synchronization was concentrated in the gamma-band, whereas activity changes were more evident in lower frequencies, which have been more reliably related to local MEG activity changes in language areas (Hirata et al., 2010; Wang et al., 2011). Our results support the view that phase coherence among gamma rhythms in distributed neural networks supports the formation of task-dependent, functionally integrated neural assemblies supporting task performance (Canolty et al., 2010), and that oscillatory phase coupling, proposed as a general mechanism underpinning functional integration within distributed brain networks (Varela et al., 2001; Fries, 2005), is relevant for inter-regional communication supporting expressive language processing. Consistent with previous findings (i.e., Doesburg et al., 2008a), we found that topographic changes in gamma-band interregional synchrony may not coincide with corresponding changes in gamma activity within the network (i.e., focal gamma increases identified using beamformer analysis).

Observed bursts of task-dependent gamma-band synchronization among regions within the expressive language network were transient, and periodically recurred at a theta rate. Similar phenomena have previously been observed during perceptual (Rodriguez et al., 1999), cognitive (Doesburg et al., 2008a), and language (Doesburg et al., 2008b) processing. Interactions between theta and gamma oscillations have been proposed to mediate the integration of local neuronal processes among distributed brain regions (von Stein and Sarnthein, 2000) and coupling between theta and gamma rhythms in human cortex have been shown to be increased in task-relevant areas during cognitive processing (Canolty et al., 2006). Gamma-band synchronization between task-activated regions is modulated by the phase of cortical theta rhythms during perception (Doesburg et al., 2009) and cognition (Doesburg et al., 2012), and several lines of evidence indicate that entrainment of gamma oscillations by theta phase may play a special role in the coordination of neuronal activity across large-scale networks (see Canolty and Knight, 2010 for review). We provide the first evidence that inter-regional gamma synchronization within language networks is modulated by theta phase, providing new insights into how task-dependent communication among spatially distributed regions is coordinated to support expressive language processing. This finding indicates that the interplay between high-frequency and low-frequency rhythms may sculpt information flow within distributed neuronal assemblies 
supporting expressive language. Specifically, these results indicate that language production involves gamma-band synchronization among task-activated cortical regions. This suggests that specific functions mediated by specialized regions integrate information supporting the performance of specific expressive language tasks by synchronizing gamma rhythms among them, producing a distributed ensemble of coherently oscillating neurons.

Low-frequency oscillations, such as those in the alpha (8$12 \mathrm{~Hz}$ ) frequency range, have been linked to inhibitory processing (see Klimesch et al., 2007). These fluctuations in inhibitory cortical activity are understood to implement "breaks" in ongoing gamma activity and to underlie observations of alpha-modulatedgamma (see Jensen and Mazaheri, 2010). Modulation of gamma activity by theta phase has also been reported (i.e., Canolty et al., 2006; Doesburg et al., 2009). Coupling of gamma oscillations to low-frequency phase likely plays a similar role for both alpha and theta frequency ranges, as task-dependent shifts of gamma coupling from alpha to theta phases have been reported (Voytek et al., 2010), suggesting that these phenomena may operate on a common continuum of neurophysiological function. Accordingly, recurrent bursts of gamma synchronization observed in the present study likely reflect multiple, successive instances of communication among activated cortical regions. As such, region pairs expressing several instances of inter-regional synchronization, such as left IFG - left MFG, are interpreted to express more iterations of inter-regional communication reflecting pronounced engagement in task-dependent increases in functional network connectivity.

Investigation of the relative frequency of instances of interregional gamma synchronizations within the activated network indicates that communication among certain activated regions is particularly pivotal for task performance. Gamma synchronization among regions was most abundant within left frontal cortex, consistent with previous observations that left frontal cortex plays a central role in expressive language. Source pairs including left inferior frontal gyrus expressed the most prolific inter-regional gamma-band synchronization, suggesting that this region plays an important role in the orchestration of information exchange throughout the activated network. The left inferior frontal gyrus is understood to be critical for expressive language, and more specifically, is vital for language tasks involving memory retrieval and selection (Thompson-Schill et al., 1998; Hirshorn and ThompsonSchill, 2006). Although some have proposed a more domain general role for left inferior frontal gyrus (see Novick et al., 2005 for review), the co-activation of left IFG in a verb generation task coincident with other areas implicated in expressive language processing (see Holland et al., 2001; Wood et al., 2004; Brown et al., 2005; Kadis et al., 2011) suggests that its engagement reflects language processing due to the influence of neural context (see McIntosh, 2000).

Cross-frequency modulation of gamma-band synchrony was most pronounced within left frontal cortex, particularly for source pairs which included the left inferior frontal gyrus. This evidences a convergence between regions expressing robust connectivity and those exhibiting cross-frequency modulation of synchrony. These results add to the burgeoning body of evidence implicating interactions among oscillations in different frequency ranges in the selection and coordinated integration of distributed neural populations into functional cell coalitions supporting cognition, perception and action (Tass et al., 1998; Burgess and Ali, 2002; Palva et al., 2005, 2010; Doesburg et al., 2009), and uniquely demonstrate that such processes are relevant for expressive language. Gamma-band synchronization between visual cortex and frontal cortex, and its modulation by theta phase, likely reflects transfer of information regarding the visually presented cue to frontal areas involved in language processing. Cross-frequency modulation of inter-hemispheric phase locking preferentially involved theta phase in left frontal cortex, further underscoring its importance in organizing information exchange among distributed co-active task-relevant regions. Taken in concert with evidence demonstrating prominent gamma synchronization in left frontal cortex these findings indicate that left frontal cortex, particularly left inferior frontal gyrus, is critical for the formation of integrated oscillatory networks supporting expressive language processing.

\section{LIMITATIONS}

We examined regional activation, and inter-regional synchronization, associated with performance of a covert verb generation task by comparing brain activity during task performance with activity recorded during a pre-stimulus baseline. Accordingly, it is not possible to distinguish which regional activations and instances of inter-regional synchrony are due to language processing and which are due to other aspects of the task based on the analyses of the present study. It is similarly not possible to determine which instances of brain activation and synchronization are related to specific aspects of language processing (e.g., semantics, phonology, etc.). Instead, interpretations regarding the putative role of regional activation and inter-regional synchronization rely on previously published literature. The present study investigated synchrony among regions showing task-dependent changes in oscillatory activity, building on previous research from our group (Doesburg et al., 2009, 2012), and others (i.e., Supp et al., 2007; Siegel et al., 2008). A limitation of this approach is that it may not detect changes in functional network connectivity which are not accompanied by changes in regional oscillatory power. Future studies employing localization techniques based on coherence or causal relations among regions (i.e., Gross et al., 2001; Michalareas et al., 2012) will be required to investigate this possibility, although our results suggest that changes in gamma synchrony are greater within the task-activated network. Beamformer source localization revealed task-dependent changes in cortical activity at low-frequencies $(5-15 \mathrm{~Hz} ; 15-25 \mathrm{~Hz})$. Although we elucidated the relationship between theta activity and inter-regional gamma synchrony, relations among low-frequency (including alpha and beta bands) increases in oscillatory power and inter-regional gamma-band connectivity remain unclear. A final limitation of our design study included the absence of acquisition of any behavioral data. The design of this study was based on blockdesign $\mathrm{fMRI}$ paradigms where response time and accuracies were not measured. In future variants of this task, aquisition of reaction time and accuracy data will allow better understanding of which neural regions are linked to particular aspects of task performance. 


\section{CONCLUSION}

The present study uniquely employed MEG to investigate oscillatory synchronization among brain regions activated during the performance of an expressive language task. We present the first evidence of task-dependent gamma-band synchronization within the expressive language network, together with the first evidence that this inter-regional synchronization is modulated by the phase of theta-band cortical oscillations. This provides novel information regarding the oscillatory mechanisms of interregional communication supporting expressive language processing. Moreover, the findings of the present study provide new insights into the relative strengths of task-dependent oscillatory

\section{REFERENCES}

Brown, T. T., Lugar, H. M., Coalson, R. S., Miezin, F. M., Petersen, S. E., and Schlaggar, B. L. (2005). Developmental changes in human cerebral functional organization for word generation. Cereb. Cortex 15, 275-290.

Burgess, A. P., and Ali, L. (2002). Functional connectivity of gamma EEG activity is modulated at low frequency during conscious recollection. Int. J. Psychophysiol. 46, 91-100.

Buschman, T. J., and Miller, E. K. (2007). Top-down versus bottom-up control of attention in the prefrontal and parietal corticies. Science 315, 1860-1865.

Calmels, C., Fourtren, M., and Stam, C. J. (2012). Beta functional connectivity modulation during the maintenance of motion information in working memory: importance of the familiarity of the visual context. Neuroscience. http://dx.doi.org/ 10.1016/j.neuroscience.2012.03.045

Canolty, R. T., Edwards, E., Dalal, S. S., Soltani, M., Nagarajan, S. S., Kirsch, H. E., Berger, M. S., Barbaro, N. M., and Knight, R. T. (2006). High gamma power is phase-locked to theta oscillations in human neocortex. Science 313, 1626-1628.

Canolty, R. T., Ganguly, K., Kennerly, S. W., Cadieu, C. F., Koepsell, K., and Wallis, J. D, Carmena, J. M. (2010). Oscillatory phase coupling coordinates anatomically dispersed functional cell assemblies. Proc. Natl. Acad. Sci. U.S.A. 107, 17356-17361.

Canolty, R. T., and Knight, R. T. (2010). The functional role of crossfrequency coupling. Trends Cogn. Sci. 14, 506-515.

Catani, M., Jones, D. K., and Ffytche, D. H. (2005). Perisylvian language networks of the human brain. Ann. Neurol. 57, 8-16.

Cheyne, D., Bakhtazad, L., and Gaetz, W. (2006). Spatiotemporal mapping of cortical activity accompanying voluntary movements using an event-related beamforming approach. Hum. Brain Mapp. 27, 213-229.

Crone, N. E., Hao, L., Hart, J. Jr., Boatman, D., Lesser, R. P., Irizarry, R., and Gordon, B. (2001). Electrocorticographic gamma activity during word production in spoken and sign language. Neurology 57, 2045-2053.

de Diego-Balaguer, R., Fuentemilla, L., and Rodriguez-Fornells, A. (2011). Brain dynamics sustaining rapid rule extraction from speech. J. Cogn. Neurosci. 23, 3105-3120.

Demiralp, T., Bayraktaroglu, Z., Lenz, D., Junge, S., Busch, N. A., Maess, B., Ergen, M., and Herrmann, C. S. (2007). Gamma amplitudes are coupled to theta phase in human EEG during visual perception. Int. J. Psychophysiol. 64, 24-30.

Doesburg, S. M., Green, J. J., McDonald, J. J., and Ward, L. M. (2009). Rhythms of consciousness: binocular rivalry reveals large-scale oscillatory network dynamics mediating visual perception. PLoS ONE 4:e6142. doi:10.1371/journal.pone.0006142

Doesburg, S. M., Green, J. J., McDonald, J. J., and Ward, L. M. (2012). Theta modulation of interregional gamma synchronization during auditory attention control. Brain Res. 1431, 77-85.

Doesburg, S. M., Kitajo, K., and Ward, L. M. (2005). Increased gammaband synchrony precedes switching of conscious perceptual objects in binocular rivalry. Neuroreport 16, 1139-1142.

Doesburg, S. M., Roggeveen, A. B., Kitajo, K., and Ward, L. M. (2008a). Large-scale gamma-band phase synchronization and selective attention. Cereb. Cortex 18, 386-396.

Doesburg, S. M., Emberson, L. L., Rahi, (2008b). Asynchrony from synchrony: long-range gamma-band neural synchrony accompanies perception of audiovisual speech A., Cameron, D., and Ward, L. M.

connectivity within the expressive language network, indicating that the left inferior frontal gyrus plays a pivotal role in network communication.

\section{ACKNOWLEDGMENTS}

This work was partially supported by a Canadian Institutes of Health Research grant (MOP-89961) to Elizabeth W. Pang as well as grants from the Ontario Brain Institute and the Hospital for Sick Children Centre for Brain and Behaviour to Sam M. Doesburg. This work was awarded first prize for a presentation given by Sarah A. Vinette at the SickKids 2011 Summer Research Program Symposium.

asynchrony. Exp. Brain Res. 185, 11-20.

Edwards, E., Nagarajan, S. S., Dalal, S. S., Canolty, R. T., Kirsch, H. E., Barbaro, N. M., and Knight, R. T. (2010). Spatiotemporal imaging of cortical activation during verb generation and picture naming. Neuroimage 50, 291-301.

Engel, A. K., König, P., and Singer, W. (1991). Direct physiological evidence for scene segmentation by temporal coding. Proc. Natl. Acad. Sci. U.S.A. 88, 9136-9140.

Ford, A., McGregor, K. M., Case, K., Crosson, B., and White, K. D. (2010). Structural connectivity of Broca's area and medial frontal cortex. $\mathrm{Neu}$ roimage 52, 1230-1237

Frey, S., Campbell, J. S., Pike, B. G., and Petrides, M. (2008). Dissociating the human language pathways with high angular resolution diffusion fiber tractography. J. Neurosci. 28, 11435-11444.

Fries, P. (2005). A mechanism for cognitive dynamics: neuronal communication through neuronal coherence. Trends Cogn. Sci. 9, 474-480.

Friederici, A. D., Brauer, J., and Lohmann, G. (2011). Maturation of the language network: from inter- to intrahemispheric connectivities. PLoS ONE 6:e20726. doi:10.1371/journal.pone.0020726.

Fukuda, M., Rothermel, R., Juhász, C., Nishida, M., Sood, S., and Asano, E. (2010). Cortical gamma-oscillations modulated by listening and overt repetition of phonemes. Neuroimage 49, 2735-2745.

Gaillard, W. D., Sachs, B. C., Whitnah, J. R., Ahmad, Z., Balsamo, L. M., Petrella, J. R., Braniecki, S. H., McKinney, C. M., Hunter, K., Xu, B., and Grandin, C. B. (2003). Developmental aspects of language processing: fMRI of verbal fluency in children and adults. Hum. Brain Mapp. 18, 176-185.

Geschwind, N. (1970). The organization of language in the brain. Science 170, 940-944.
Golfinopoulos, E., Tourville, J. A., and Guenther, F. H. (2010). The integration of large-scale neural network modeling and functional brain imaging in speech motor control. Neuroimage 52, 862-874.

Gray, C. M., König, P., Engel, A. K., and Singer, W. (1989). Oscillatory responses in cat visual cortex exhibit inter-columnar synchronization which reflects global stimulus properties. Nature 338, 334-337.

Gray, C. M., and Singer, W. (1989). Stimulus-specific neuronal oscillations in orientation columns of cat visual cortex. Proc. Natl. Acad. Sci. U.S.A. 86, 1698-1702

Gregoriou, G. G., Gotts, S. J., Zhou, H., and Desimone, R. (2009). High-frequency, long-range coupling between prefrontal and visual cortex during attention. Science 324, 1207-1210.

Gross, J., Kujala, J., Hamalainen, M., Timmermann, L., and Schnitzler, A. (2001). Dynamic imaging of coherent sources: studying neural interactions in the human brain. Proc. Natl. Acad. Sci. U.S.A. 98, 694-699.

Hickok, G., and Poeppel, D. (2004). Dorsal and ventral streams: a framework for understanding aspects of the functional anatomy of language. Cognition 92, 67-99.

Hillebrand, A., Barnes, G. R., Bosboom, J. L., Berendese, H. W., and Stam, C. J. (2012). Frequency-dependent functional connectivity within restingstate networks: an atlas-based MEG beamformer solution. Neuroimage 15, 3909-3921.

Hirata, M., Tetsu, G., Barnes, G., Unekawa, Y., Yanagisawa, T., Kato, A, Oshino, S., Kishima, H., Hashimoto, N., Saitoh, Y., Tani, N., Yorifuji, S., and Yoshimine, T. (2010). Language dominance and mapping based on neuromagnetic oscillatory changes: comparison with invasive procedures. J. Neurosurg. 112, 528-538. 
Hirshorn, E. A., and Thompson-Schill, S. L. (2006). Role of the left inferior frontal gyrus in covert word retrieval: neural correlates of switching during verbal fluency. Neuropsychologia 44, 2547-2557.

Holland, S. K., Plante, E., Byars, A. W., Strawsburg, R. H., Schmithorst, V. J., and Ball, W. S. (2001). Normal fMRI brain activation patterns in children performing a verb generation task. Neuroimage 14, 837-843.

Indefrey, P., and Levelt, W. J. (2004). The spatial and temporal signatures of word production components. Cognition 92, 101-144

Jensen, O., and Mazaheri, A. (2010). Shaping functional architecture by oscillatory alpha activity: gating by inhibition. Front. Hum. Neurosci. 4:186. doi:10.3389/fnhum.2010.00186

Jerbi, K., Ossandón, T., Hamamé, C. M., Senova, S., Dalal, S. S., Jung, J., Minotti, L., Bertrand, O., Berthoz, A., Kahane, P., and Lachaux, J. P. (2009). Task-related gamma-band dynamics from an intracerebral perspective: review and implications for surface EEG and MEG. Hum. Brain Mapp. 30, 896-904.

Kadis, D. S., Pang, E. W., Mills, T., Taylor, M. J., McAndrews, M. P., and Smith, M. L. (2011). Characterizing the normal developmental trajectory of expressive language lateralization using magnetoencephalography. J. Int. Neuropsychol. Soc. 17, 896-904.

Kelly, C., Uddin, L. Q., Shehzad, Z., Margulies, D. S., Castellanos, F. X., Milham, M. P., and Petrides, M. (2010). Broca's region: linking human brain functional connectivity data and non-human primate tracing anatomy studies. Eur. J. Neurosci. 32, 383-398.

Khader, P., and Rösler, F. (2004). EEG power and coherence analysis of visually presented nouns and verbs reveals left frontal processing differences. Neurosci. Lett. 354, 111-114.

Khader, P. H., Jost, K., Mertens, M., Bien, S., and Rösler, F. (2010). Neural correlates of generating visual nouns and motor verbs in a minimal phrase context. Brain Res. 1318, 122-132.

Klimesch, W., Sauseng, P., and Hansylmayer, S. (2007). EEG alpha oscillations: the inhibition-timing hypothesis. Brain Res. Rev. 53, 63-88.

Korzeniewska, A., Franaszczuk, P. J., Crainiceanu, C. M., Kus, R., and Crone, N. E. (2011). Dynamics of large-scale cortical interactions at high gamma frequencies during word production: event related causality (ERC) analysis of human electrocorticography (ECoG). Neuroimage 56, 2218-2237.

Koyama, M. S., Di Martino, A., Zuo, XN., Kelly, C., Mennes, M., Jutagir, D. R., Castellanos, F. X., and Milham, M. P. (2011). Resting-state functional connectivity indexes reading competence in children and adults. J. Neurosci. 31, 8617-8624.

Lachaux, J. P., Rodriguez, E., Martinerie, J., and Varela, F. J. (1999). Measuring phase synchrony in brain signals. Hum. Brain Mapp. 8, 194-208.

Levelt, W. J., Roelofs, A., and Meyer, A. S. (1999). A theory of lexical access in speech production. Behav. Brain Sci. 22, 1-38.

Llinás, R., Urbano, F. J., Leznik, E., Ramírez, R. R., and van Marle, H. J. F. (2005). Rhythmic and dysrhythmic thalamocortical dynamics: GABA systems and the edge effect. Trends Neurosci. 28, 325-333.

Mazziotta, J. C., Toga, A., Evans, A., Fox, P., Lancaster, J., Zilles, K., Simpson, G., Woods, R., Paus, T., Pike, B., Holmes, C., Collins, L., Thompson, P., MacDonald, D., Schormann, T., Amunts, K., Palomero-Gallagher, N., Parsons, L., Narr, K., Kabani, N., LeGoualher, G., Boomsma, D., Cannon, T., Kawashima, R., and Mazoyer, B. (2001). A probabilistic atlas and reference system for the human brain. Philos. Trans. R. Soc. Lond. B Biol. Sci. 356, 1293-1322.

Mazziotta, J. C., Toga, A. W., Evans, A., Lancaster, J. L., and Fox, P. T. (1995). A probabilistic atlas of the human brain: theory and rational for its development. Neuroimage 2, 89-101.

McIntosh, A. R. (2000). Towards a network theory of cognition. Neural. Netw. 13, 861-870.

Melloni, L., Molina, C., Pena, M., Torres, D., Singer, W., and Rodriguez, E. (2007). Synchronization of neural activity across cortical areas correlates with conscious perception. J. Neurosci. 27, 2858-2865.

Michalareas, G., Schloffelen, J. M., Paterson, G., and Gross, J. (2012). Investigating causality between interacting brain areas with multivariate auroregressive models of MEG sensor data. Hum. Brain Mapp. doi:10.1002/hbm.21482.

Novick, J. M., Trueswell, J. C., and Thompson-Schill, S. L. (2005). Cognitive control and parsing: Reexamining the role of Broca's area in sentence comprehension. Cogn. Affect. Behav. Neurosci. 5, 263-281.

Oldfield, R. C. (1971). The assessment and analysis of handedness: the Edinburgh inventory. Neuropsychologia 9, 97-113.
Palva, J. M., Monto, S., Kulashekhar, S., and Palva, S. (2010). Neuronal synchrony reveals working memory networks and predicts individual memory capacity. Proc. Natl. Acad. Sci. U.S.A. 107, 7580-7585.

Palva, J. M., Palva, S., and Kaila, K. (2005). Phase synchrony among neuronal oscillations in the human cortex. J. Neurosci. 25, 3962-3972.

Pang, E. W., Wang, F., Malone, M., Kadis, D. S., and Donner, E. J. (2011). Localization of Broca's area using verb generation tasks in the MEG: validation against fMRI. Neurosci. Lett. 490, 215-219.

Pei, X,. Leuthardt, E. C., Gaona, C. M., Brunner, P., Wolpaw, J. R., and Schalk, G. (2011). Spatiotemporal dynamics of electrocorticographic high gamma activity during overt and covert word repetition. $\mathrm{Neu}$ roimage 54, 2960-2972.

Perani, D., Cappa, S. F., Schnur, T., Tettamanti, M., Collina, S., Rosa, M. M., and Fazio, F. (1999). The neural correlates of verb and noun processing: a PET study. Brain 122, 2337-2344.

Price, C. J. (2010). The anatomy of language: a review of $100 \mathrm{fMRI}$ studies published in 2009. Ann. N.Y. Acad. Sci. 119, 62-88.

Robinson, S. E., and Vrba, J. (1999). "Functional neuroimaging by synthetic aperture magnetometry (SAM)," in Recent Advances in Biomagnetism, eds T. Yoshimoto, M. Kotani, S. Kuriki, H. Karibe, and N. Nakasato (Sendai: Tohoku University Press), 302-305.

Rodriguez, E., George, N., Lachaux, J. P., Martinerie, J., Renault, B., and Varela, F. J. (1999). Perception's shadow: long-distance synchronization of human brain activity. Nature 397, 430-433.

Rose, M., and Büchel, C. (2005). Neural coupling binds visual tokens to moving stimuli. J. Neurosci. 25, 10101-10104.

Rose, M., Sommer, T., and Büchel, C. (2006). Integration of local features to a global percept by neural coupling. Cereb. Cortex 16, 1522-1528.

Roux, F. E., Boulanouar, K., Lotterie, J. A., Mejdoubi, M., LeSage, J. P., and Berry, I. (2003). Language functional magnetic resonance imaging in preoperative assessment of language areas: correlation with direct cortical stimulation. Neurosurgery 52, 1335-1345.

Salmelin, R. (2007). Clinical neurophysiology of language: the MEG approach. Clin. Neurophysiol. 118, 237-254.
Sarnthein, J, Petsche, H., Rappelsberger, P., Shaw, G., and von Stein, A. (1998). Synchronization between prefrontal and posterior association cortex during human working memory. Proc. Natl. Acad. Sci. U.S.A. 95, 7092-7096.

Saur, D., Kreher, B. W., Schnell, S., Kummerer, D., Kellmeyer, P., Vrys, M. S. Umarova, R., Musso, M., Glauche, V., Abel, S., Huber, W., Riinties, M. Hennig, J., and Weiller, C. (2008). Ventral and dorsal pathways for language. Proc. Natl. Acad. Sci. U.S.A. 105, 18035-18040.

Sauseng, P., Klimesch, W., Gruber, W. R., and Birbaumer, N. (2008). Cross-frequency phase synchronization: a brain mechanism of memory matching and attention. Neuroimage 40, 308-317.

Schack, B., Vath, N., Petsche, H., Geissler, H. G., and Moller, E. (2002). Phasecoupling of theta-gamma EEG rhythms during short-term memory processing. Int. J. Psychophysiol. 44, 143-163.

Schuhmann, T., Schiller, N. O., Goebel, R., and Sack, A. T. (2012). Speaking of which: dissecting the neurocognitive network of language production in picture naming. Cereb. Cortex 22, 701-709.

Sekihara, K., Nagarajan, S., Poeppel, D., Marantz, A., and Miyashita, Y. (2001). Reconstructing spatiotemporal activities of neural sources using an MEG vector beamformer technique. IEEE Trans. Biomed. Eng. 48, 760-771.

Shapiro, K. A., Moo, L. R., and Caramazza, A. (2006). Cortical signatures of noun and verb production. Proc. Natl. Acad. Sci. U.S.A. 103, 1644-1649.

Siegel, M., Donner, T. H., Oostenveld, R., Fries, P., and Engel, K. (2008). Neuronal synchronization along the dorsal visual pathway reflects the focus of spatial attention. Neuron 60 , 709-719.

Stam, C. J., Nolte, G. N., and Daffertshofer, A. (2007). Phase lag index: assessment of functional connectivity from multi channel EEG and MEG with diminished bias from common sources. Hum. Brain Mapp. 28, 1178-1193.

Supp, G. G., Schögl, A., Trujillo-Barreto, N., Müller, M. M., and Gruber, T. (2007). Directed cortical information flow during human object recognition: analyzing induced EEG gamma-band responses in brain's source space. PLoS ONE 2:e684. doi:10.1371/journal.pone.0000684 Szaflarski, J. P., Holland, S. K., Jacola, L. M., Lindsell, C., Privitera, M. D., 
and Szaflarski, M. (2008). Comprehensive presurgical functional MRI language evaluation in adult patients with epilepsy. Epilepsy Behav. 12, 74-83.

Tass, P., Rosenblum, M. G., Weule, J., Pikovsky, A., Volmann, J., Schnitzler, A., and Freund, H. -J. (1998). Detection of n:m phase locking in noisy data: application to magnetoencephalography. Phys. Rev. Lett. 81, 3291-3294.

Thompson-Schill, S. L., Swick, D., Farah, M. J., D’Esposito, M., Kan, I. P., and Knight, R. T. (1998). Verb generation in patients with focal frontal lesions: a neuropsychological test of neuroimaging findings. Proc. Natl. Acad. Sci. U.S.A. 26, 14792-14797.

Tomasi, D., and Volkow, N. D. (2012). Resting functional connectivity of language networks: characterization and reproducibility. Mol. Psychiatry. doi: 10.1038/mp.2011.177

Turken, A. U., and Dronkers, N. F. (2011). The neural architecture of the language comprehension network: converging evidence from lesion and connectivity analyses. Front. Syst. Neurosci. 5, 1-20. doi: 10.3389/fnsys.2011.00001
Uhlhaas, P. J., Pipa, G., Lima, B., Melloni, L., Neuenschwander, S., Nicolic, D., and Singer, W. (2009). Neural synchrony in cortical networks: history, concept and current status. Front. Integr. Neurosci. 3:17. doi:10.3389/neuro.07.017.2009

Van Veen, B. D., van Drongelen, W., Yuchtman, M., and Suzuki, A. (1997). Localization of brain electrical activity via linearly constrained minimum variance spatial filtering. IEEE Trans. Biomed. Eng. 44, 867-880.

Varela, F., Lachaux, J. P., Rodriguez, E., and Martinerie, J. (2001). The brainweb: phase synchronization and large-scale integration. Nat. Rev. Neurosci. 2, 229-239.

von Stein, A., and Sarnthein, J. (2000). Different frequencies for different scales of cortical integration: from local gamma to long range alpha/theta synchronization. Int. J. Psychophsiol. 38, 301-313.

Voytek, B., Canolty, R. T., Shestyuk, A. Crone, N. E., Parvizi, J., and Knight, R. T. (2010). Shifts in gamma phaseamplitude coupling frequency from theta to alpha over posterior cortex during visual tasks. Front. Hum. Neurosci. 4, 191.
Vrba, J., and Robinson, S. E. (2001). Signal processing in magnetoencephalography. Methods (Duluth), 25, 249-271.

Wang, Y., Xiang, J., Vannest, J., Holroyd, T., Narmoneva, D., Horn, P., Liu, Y., Rose, D., and deGrauw, T, Holland, S. (2011). Neuromagnetic measures of word processing in bilinguals and monolinguals. Clin. Neurophysiol. 112, 1706-1717.

Ward, L. M. (2003). Synchronous neural oscillations and cognitive processes. Trends Cogn. Sci. (Regul. Ed.) 7 553-559.

Weiss, S., Mueller, H. M., Schack, B., King, J. W., Kutas, M., and Rappelsberger, P. (2005). Increased neuronal communication accompanying sentence completion. Int. J. Psychophysiol. 57, 129-141.

Womelsdorf, T., Schoffelen, J. M. Oostenveld, R., Singer, W., Desimone, R., Engel, A. K., and Fries, P. (2007). Modulation of neuronal interactions through neuronal synchronization. Science 316, 1609-1612.

Wood, A. G., Harvey, A. S., Wellard, R. M., Abbott, D. F., Anderson, V., Kean,
M., Saling, M. M., and Jackson, G. D. (2004). Language cortex activation in normal children. Neurology 63, 1035-1044.

Conflict of Interest Statement: The authors declare that the research was conducted in the absence of any commercial or financial relationships that could be construed as a potential conflict of interest.

Received: 05 January 2012; accepted: 25 May 2012; published online: 13 June 2012.

Citation: Doesburg SM, Vinette SA, Cheung MJ and Pang EW (2012) Thetamodulated gamma-band synchronization among activated regions during a verb generation task. Front. Psychology 3:195. doi: 10.3389/fpsyg.2012.00195

This article was submitted to Frontiers in Language Sciences, a specialty of Frontiers in Psychology.

Copyright (c) 2012 Doesburg, Vinette, Cheung and Pang. This is an open-access article distributed under the terms of the Creative Commons Attribution Non Commercial License, which permits noncommercial use, distribution, and reproduction in other forums, provided the original authors and source are credited. 